\title{
A New Agile Radiating System Called Electromagnetic Band Gap Matrix Antenna
}

\author{
Hussein Abou Taam, ${ }^{1}$ Moustapha Salah Toubet, ${ }^{1}$ Thierry Monediere, \\ Bernard Jecko, ${ }^{1}$ and Mohammad Rammal ${ }^{2}$ \\ ${ }^{1}$ OSA Department, XLIM, UMR CNRS 7252, 87000 Limoges, France \\ ${ }^{2}$ GRIT Department, IUT, Lebanese University, Saida, Lebanon \\ Correspondence should be addressed to Hussein Abou Taam; hk_doc@hotmail.com
}

Received 16 October 2013; Revised 20 February 2014; Accepted 24 February 2014; Published 25 March 2014

Academic Editor: Xiuping Li

Copyright ( 2014 Hussein Abou Taam et al. This is an open access article distributed under the Creative Commons Attribution License, which permits unrestricted use, distribution, and reproduction in any medium, provided the original work is properly cited.

Civil and military applications are increasingly in need for agile antenna devices which respond to wireless telecommunications, radars, and electronic warfare requirements. The objective of this paper is to design a new agile antenna system called electromagnetic band gap (EBG) matrix. The working principle of this antenna is based on the radiating aperture theory and constitutes the subject of an accepted CNRS patent. In order to highlight the interest and the originality of this antenna, we present a comparison between it and a classical patch array only for the (one-dimensional) $1 \mathrm{D}$ configuration by using a rigorous full wave simulation (CST Microwave software). In addition, EBG matrix antenna can be controlled by specific synthesis algorithms. These algorithms use inside their; optimization loop an analysis procedure to evaluate the radiation pattern. The analysis procedure is described and validated at the end of this paper.

\section{Introduction}

A generic EBG antenna consists of a cavity created by a frequency selective surface (FSS) at the top and a metallic ground plane at the bottom. The energy is coupled to the cavity using a fed antenna such as a dipole, slot, or patch $[1,2]$. The EBG antenna has aroused a growing interest of researchers in the last few years due to its capacity to enhance the directivity of a single source, its potentiality in beam forming, its dual-band frequency, bandwidth enhancement, and its polarization diversity [3-6]. Among the papers found in different literatures, a recent study presented in [7] showed that it is possible to limit the radiating aperture of an EBG antenna by inserting 2 metallic walls (along a single direction) in order to obtain an elliptical radiating aperture. Therefore, the proposed idea in this paper is to limit the radiating aperture by inserting 4 metallic walls (in two perpendicular directions) at the antenna's edges in order to obtain a homogenous square aperture. This new structure is called "Pixel" and constitutes the elementary antenna of the EBG matrix.

The aim of this work is to present an EBG matrix design. The working principle of this antenna constitutes the subject of an accepted CNRS patent [8]. The EBG matrix is based on the radiating aperture theory which is not the case in different array concept that is based on an ordinary array law. In order to highlight the interest and the originality of the EBG matrix, a comparison is made between a $1 D$ EBG matrix and a linear classical antenna array (e.g., patch array). This comparison shows that the EBG matrix is a loosely coupled system and it is very efficient for beam steering applications.

In addition, the paper shows that the agile EBG matrix can be controlled by using specific algorithms in order to respond to imposed radiation objectives. These algorithms use inside their optimization loop an analysis procedure to evaluate the radiation patterns, for each iteration. The analysis procedure is described and validated in order to verify the EBG matrix working principle.

\section{Working Principle of the Antenna}

The EBG matrix works similarly to a radiating aperture antenna. The concept is based on generating a radiating surface for any desired shape. Based on the radiating aperture 


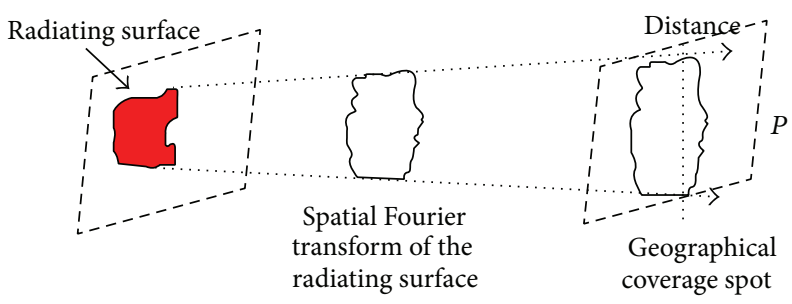

FIGURE 1: Schematic representation of the radiating aperture antenna working principle.

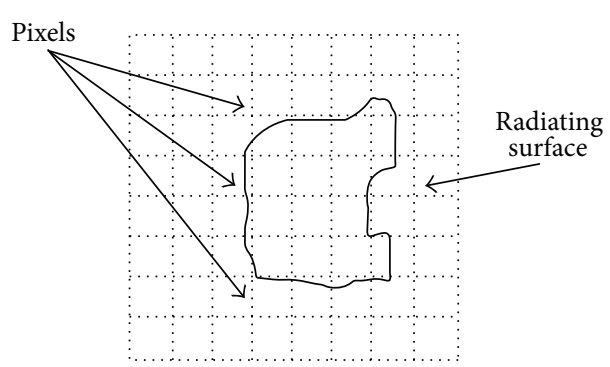

(a)

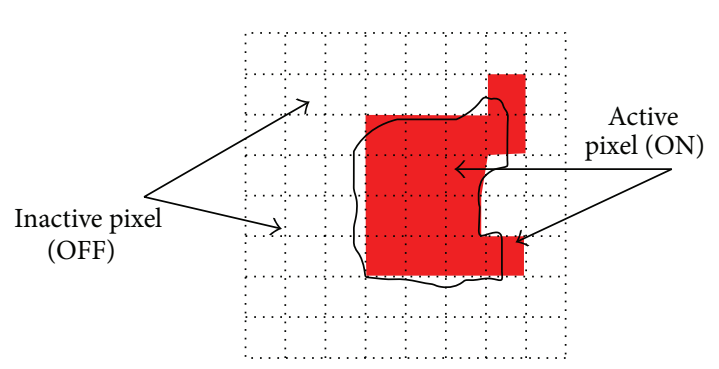

(b)

FIGURE 2: (a) Radiating surface pixilated by the antenna elements (b) and generation of the desired radiating surface.

theory, this radiating surface creates radiation patterns to ensure a given geographical coverage (Figure 1). To recall, the general analytical equation of the radiated electric field obtained at an observation point $P$ of the space from a radiating surface is presented in (1). Equation (2) looks like a spatial Fourier transform which transforms the $(x, y)$ coordinates of the near electric field distribution $E_{s}(x, y)$ existing on the radiating surface into $(\theta, \varphi)$ ones on the space at the point $P$ (equivalent principle).

Indeed, the desired radiating surface must be pixilated by introducing elementary antenna pixels which form a planar matrix antenna (Figure 2(a)). The desired radiating surface is built by activating the corresponding pixels as shown in Figure 2(b). In the simple case, the pixels are fed using only switch devices (ON State: red pixels and OFF State: white pixels). However, in a sophisticated case, each pixel can be fed by given weights in amplitude and phase using variable amplifiers and variable phase shifters. Consider the following:

$$
\begin{gathered}
\vec{E}(p)=\frac{j k}{4 \pi} \psi(R)(1+\cos \theta)\left(\cos \varphi \overrightarrow{e_{\theta}}-\sin \varphi \overrightarrow{e_{\varphi}}\right) F \\
F=\oiint_{s} E_{s}(x, y) e^{j(k x \sin \theta \cos \varphi+k y \sin \theta \sin \varphi)} d s .
\end{gathered}
$$

In order to generate any desired shape of the radiating surface, it is very advantageous to have an antenna system which is formed by special radiating elements. These elements, according to their states, should give a good approximation of the desired radiating surface. Indeed, each element must generate a square and quasi-uniform radiating aperture which is limited to the element's dimensions. Therefore, an EBG antenna called "Pixel" is designed and presented in the next section.

\section{Elementary Pixel}

The studied EBG elementary antenna is similar to a classical EBG antenna acting as a resonant cavity formed between the ground plane and the frequency selective surface (FSS) placed above it in the $z$ direction $[1,2]$, as shown in Figure 3. It usually generates on its roof a circular radiating aperture as shown in Figure 3(c). The feeding system is constituted of a patch placed above the dielectric substrate (permittivity = 4.4) and located at the EBG antenna center.

The aim is to confine the electric field distribution in the cavity, in order to modify the circular radiating aperture into a homogenous square one. To carry this out, four vertical metallic walls are inserted in the resonant cavity at the edges (Figure 4(a)). This is possible due to the radial evanescent mode existing in the EBG cavity prohibiting thereby any catastrophic transverse resonance. A square radiating aperture is obtained with a good uniformity (Figure 4(b)). The EBG cavity with metallic walls is called "pixel" and constitutes the elementary antenna of the EBG matrix which is presented in the next section.

\section{Conception of EBG Matrix}

The proposed idea of designing an EBG matrix is to associate several joint identical pixels along two directions to form 2D EBG matrix or along a single direction to form $1 \mathrm{D} \mathrm{EBG}$ matrix. This approach is illustrated in Figures 5(a) and 5(b) that show, respectively, $2 \mathrm{D}$ EBG matrix formed by $N * M$ pixels and $1 \mathrm{D}$ EBG matrix formed by $N * 1$ pixels.

Therefore, two designed configurations are presented for the EBG matrix antenna. They can be used to form any desired shape for the radiating surface corresponding to an imposed radiation pattern for geographical coverage 


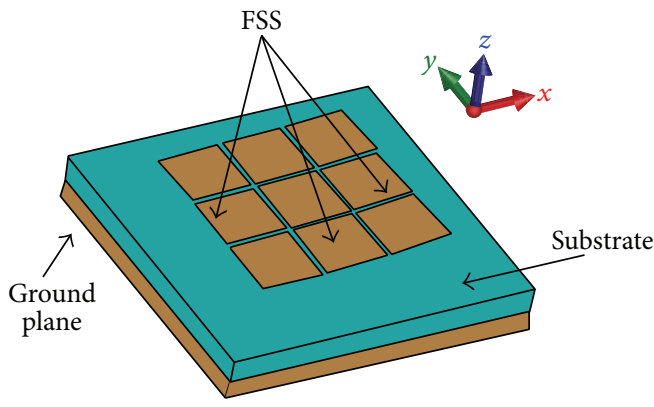

(a)

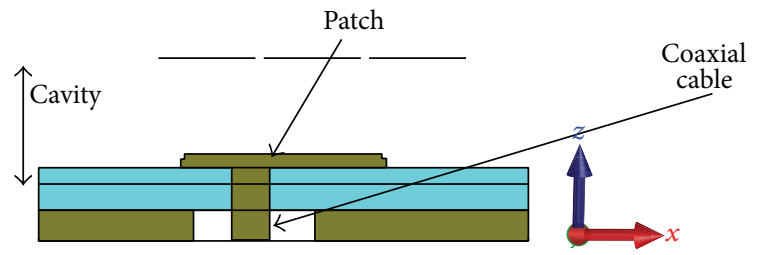

(b)
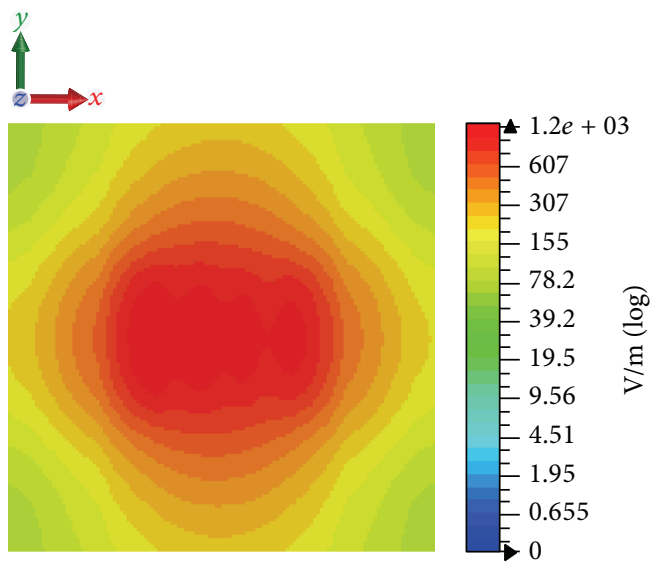

(c)

FIGURE 3: Elementary EBG antenna, perspective view (a), inside view (cut-plane at the middle along (0y)) (b), and associated near electric field distribution $E_{s}(x, y)(\mathrm{c})$.

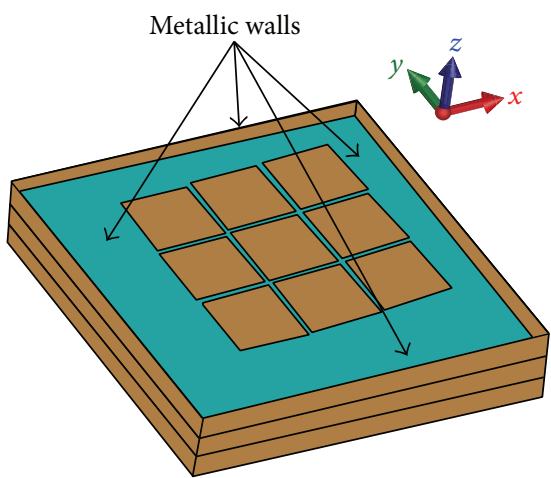

(a)
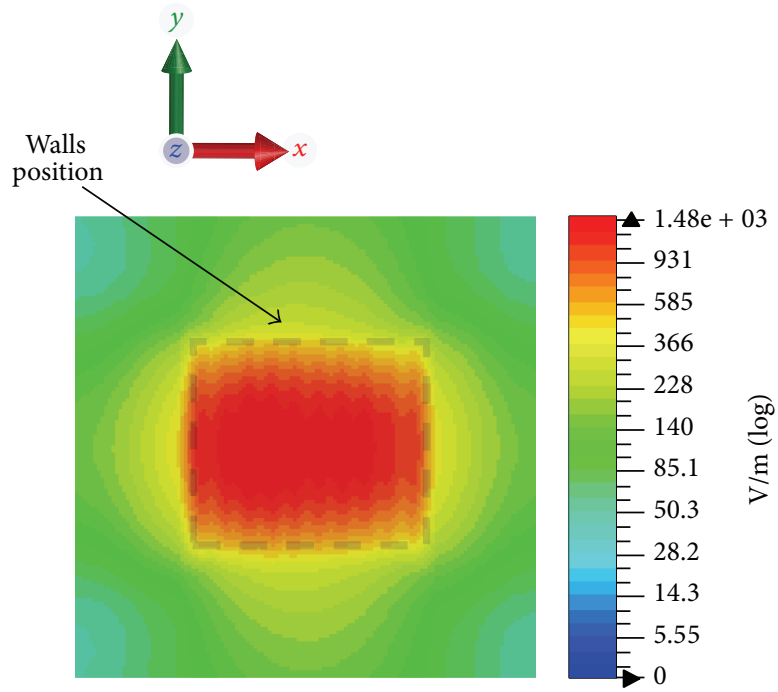

(b)

FIGURE 4: Elementary EBG antenna with walls (a) and associated square radiating aperture with quasi-uniform electric field distribution $E_{s}(x, y)(\mathrm{b})$. 


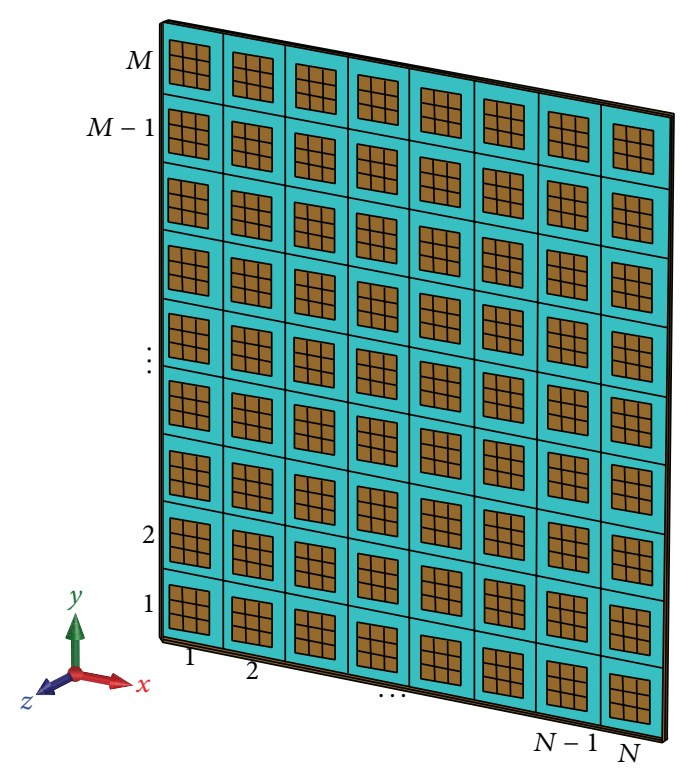

(a)

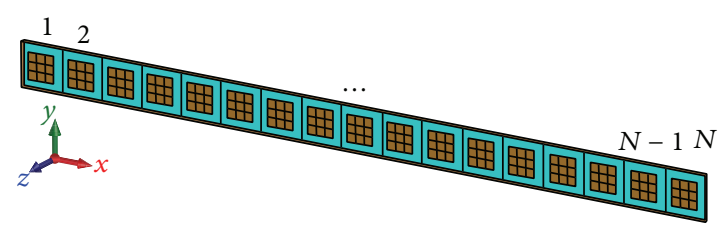

(b)

FIgURE 5: 2D EBG matrix formed by $N * M$ pixels (a) and 1D EBG matrix formed by $N * 1$ pixels (b).

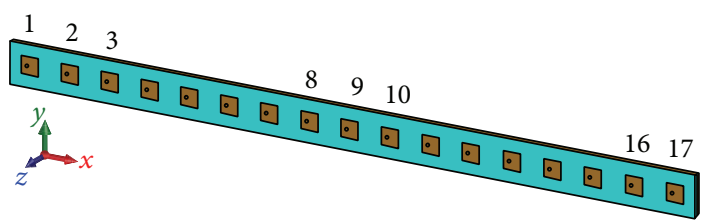

Figure 6: Patch array formed by 17 elements spaced by $0.5 \lambda$.

application. Nevertheless, this paper presents a study on the second configuration (Figure 5(b)) in order to highlight the interest and the originality of the EBG matrix, especially for beam steering applications. A comparison is made between a 1 D EBG matrix $(N=17$; element spacing $=0.5 \lambda)$ and a classical linear array (e.g., patch array).

\section{Special Radiating Surface for EBG Matrix}

For a linear patch array (Figure 6) which presents the same elements number (17 elements) and the same element spacing $(0.5 \lambda)$ of the 1D EBG matrix (Figure 5(b)), the elementary radiating aperture overlaps the neighboring elements, which induces a significant mutual coupling. The originality of the $1 \mathrm{D}$ EBG matrix is to limit each elementary radiating aperture at the pixel's dimensions in order not to disturb the neighboring pixels [9]. In addition, the formed radiating aperture is square, equiamplitude, and equiphase along the pixel's dimensions (Figure 7(b)), which is not the case for the patch array (Figure 7(a)). A global uniform radiating surface (equiamplitude and equiphase) is formed by the association of the jointed and limited elementary radiating apertures when all the pixels are fed (Figure 7(c)). 1D EBG matrix does not act like a classical array but it is considered a special radiating surface. Noting that, the radiation pattern can be practically obtained, by a spatial Fourier transform (1) of the radiating surface, according to the radiating aperture theory.

The first observation which appears in the 1D EBG matrix is the weak mutual coupling between neighboring pixels for small element spacing [9]. Indeed, the presence of metallic walls at the pixel's edges and the presence of the EBG mode inside each pixel's cavity insure a good isolation between neighboring pixels, making them independent from each other. The feeding of the central element (number 9) of the two structures gives us the matching coefficients of the central element (Figure 8(a)) and the mutual coupling level with the neighboring ones (Figure $8(\mathrm{~b})$ ). In addition, the obtained results are compared with a $1 \mathrm{D}$ array of cavity-backed patches to illustrate the effect of the EBG mode inside the pixel's cavity. The results show that the mutual coupling level for the EBG matrix is much better than the other structures. The mutual coupling level for the EBG matrix is less than $-24 \mathrm{~dB}$ at the working frequency of $8 \mathrm{GHz}$.

In the next section, we present an important property which appears in $1 \mathrm{D}$ EBG matrix, which is the possibility to achieve a good beam steering along a wide angular range.

\section{Beam Steering with EBG Matrix}

In order to highlight the advantage of the EBG matrix in the beam steering application, a comparison is made between 


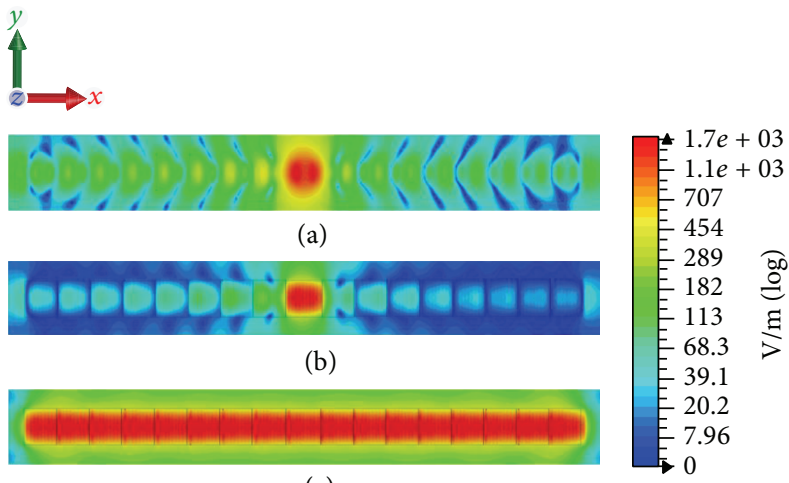

(c)

FIGURE 7: Cartography of electric field distribution: (a) central patch is fed; (b) central EBG pixel is fed; (c) all EBG pixels of the matrix are fed.

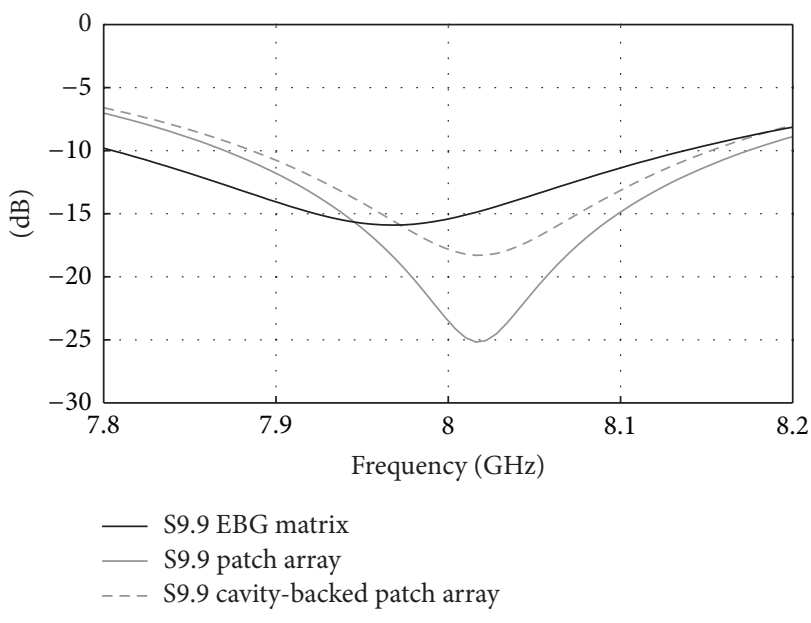

(a)

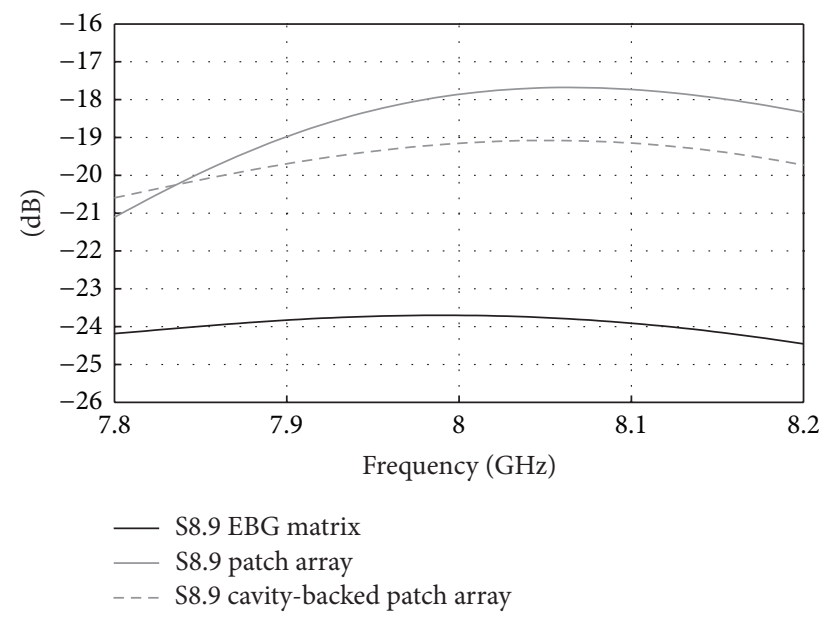

(b)

FiguRE 8: (a) Matching coefficients of the central element (b) and coupling coefficients between the central element and the first left neighboring.

the two structures (EBG matrix and patch array) already presented in the previous section. The main idea is to show that the $1 D$ EBG matrix is an efficient system for high scanning angles.

Figure 9(a) presents the radiation patterns of the two structures when each radiating element is fed in equiamplitude and equiphase. In this case, quasi-identical radiation patterns are obtained with the same gain of $18 \mathrm{~dB}$ at the broadside direction due to the fact that the two structures have the same dimensions. If the main lobes are steered to a high angle of $70^{\circ}$, the results are different (Figure 9(b)). The maximum gain is more conserved with the EBG matrix than the patch array. Indeed, the gap of the gain between the ones obtained for $\theta$ equal to $0^{\circ}$ and $\theta$ equal to $70^{\circ}$ is $3 \mathrm{~dB}$ for the $1 \mathrm{D}$ EBG matrix and $7 \mathrm{~dB}$ for the patch array. Concerning the side lobe levels, they are more reduced for the 1D EBG matrix for beam steering case (Figure 9(b)). In order to be more explicit and in the case of EBG matrix, the side lobes levels (SLL) are lower than $11 \mathrm{~dB}$ relatively to the maximum gain. However, in the case of patch array, the SLL are lower than $7 \mathrm{~dB}$ relatively to the maximum gain.
Finally, the back radiation is also reduced with a $1 \mathrm{D}$ EBG matrix. It is, respectively, lower than $13 \mathrm{~dB}$ and $6 \mathrm{~dB}$, relatively to the maximum gain for the EBG matrix and the patch array. All these performances are due to the radiating surface magnitude of the EBG matrix which is less degraded than the patch array design after beam steering, because of the isolation between the pixels and the better conservation of the radiating surface efficiency obtained at the broadside direction which is already presented in Figure 7(c).

Thus, Figure 10 presents the maximum gain value evolution versus the scanning angle value for the two structures to fully understand this advantage. These results show a better conservation of the maximum gain for the EBG matrix regardless of the scanning angle, and more specifically for high scanning angles.

\section{Analysis Procedure}

As all agile antenna systems, the EBG matrix antenna can be controlled by using specific synthesis algorithms which will enable the antenna radiation to follow imposed radiation 


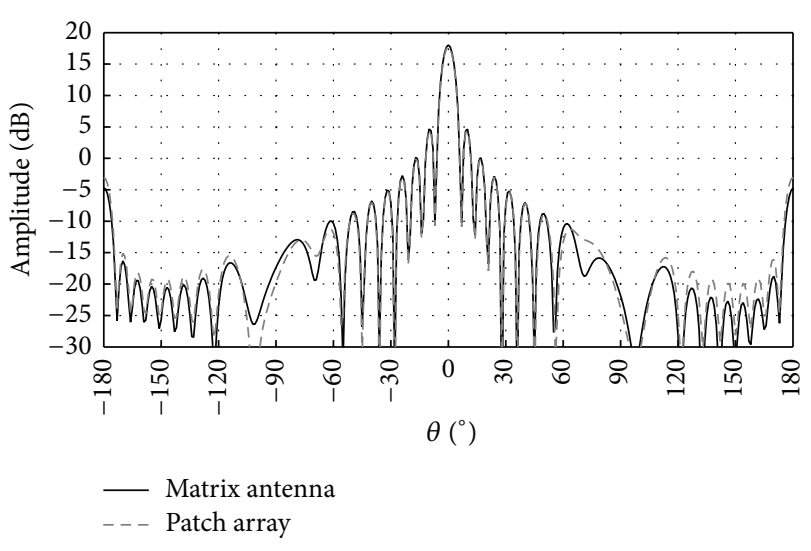

(a)

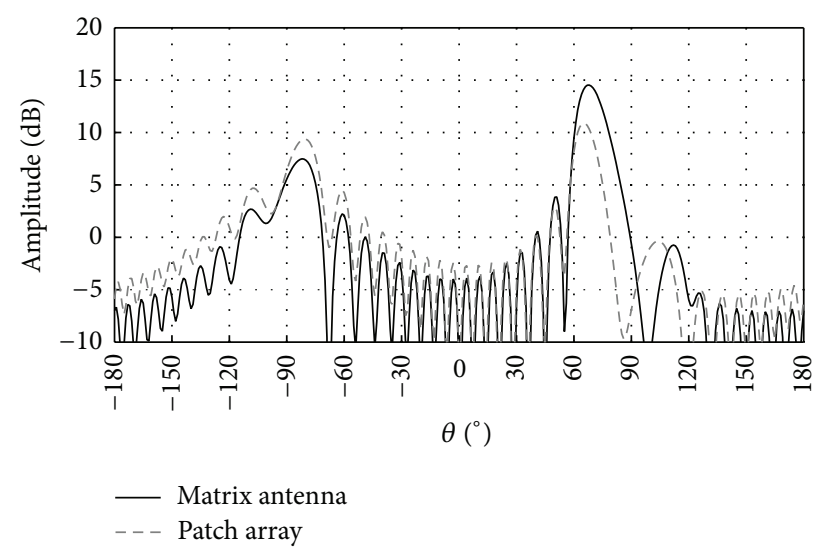

(b)

FIGURE 9: Comparison of radiation patterns between EBG matrix and patch array (a) at the broadside direction and (b) after beam steering of $70^{\circ}$.

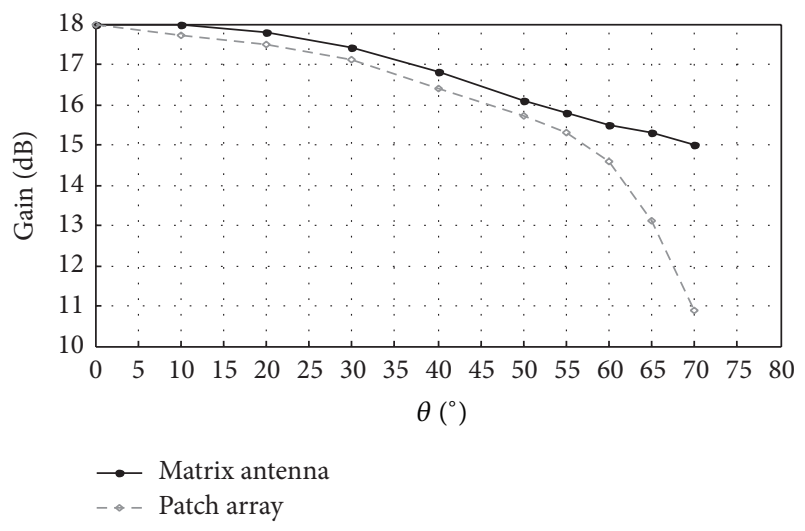

FIGURE 10: Evolution of the maximum gain value versus scanning angle value.

patterns. In the optimization loop of these algorithms, an analysis procedure is necessary to evaluate the radiation pattern, for each iteration. However, it is not possible to use rigorous simulation software inside the synthesis loop due to the time burden. Therefore, it will be necessary to model an efficient analysis tool to replace the rigorous simulation software.

The analysis procedure is based on the radiating aperture theory. The radiation patterns are calculated, according to (1) and (2), from the near electric field distribution $E_{s}(x, y)$ existing on the radiating surface. Indeed, the originality of the EBG matrix is that each pixel generates a uniform radiating aperture isolated and independent from the neighboring ones as in Figure 7(b) leading to obtaining of the same elementary radiating apertures $E_{s}(x, y)$ regardless of the pixel position. The analysis procedure is modeled by using Matlab software. The analysis code imports the near electric field distribution $E_{s}(x, y)$ from one pixel (obtained by rigorous full wave simulation once for all) and evaluates the global radiation pattern of the EBG matrix taking into consideration the weights applied on the input pixels.
Therefore, in order to validate the analysis procedure, a comparison is made between results calculated by the analysis code and others obtained by a rigorous full wave simulation using CST Microwave software. Figure 11 shows two cases of figures (broadside direction in Figure 11(a) and steered direction at $-50^{\circ}$ in Figure 11(b)). A perfect agreement is obtained except for the back radiation area due to the fact that the analytical code cannot consider the diffraction at structure edges. However, we would like to mention that the analysis procedure is described and validated but the synthesis algorithm constitutes a future perspective.

\section{Conclusion}

In this paper, a new antenna system called EBG matrix is presented. The principle of this antenna constitutes the subject of an accepted CNRS patent [8]. The originality is the special radiating aperture formed by each pixel of the matrix. The radiating aperture of each pixel is square, equiamplitude, and equiphase regardless of its position in the EBG matrix.

The fact that the radiating aperture is confined in the pixel's cavity and does not run over the pixel's dimensions insures isolation between neighboring elements. Moreover, the later isolation leads to obtaining a low mutual coupling in the EBG matrix making this antenna system more advantageous than the classical antenna array. In addition to that, this paper shows that $1 \mathrm{D}$ EBG matrix is a very efficient system for high steering angles, because the main lobe gain is more conserved with accepted side lobes and back radiation levels, making the antenna system more flexible.

The EBG matrix can be controlled by specific synthesis algorithms which use inside their optimization loop an analysis procedure to evaluate the radiation pattern for each iteration. The proposed analysis procedure is based on the radiating aperture theory. It is described and validated in this paper. The synthesis algorithm constitutes for us a future perspective.

Finally, due to the complexity, the number and the cost of the structures considered in this paper were not 


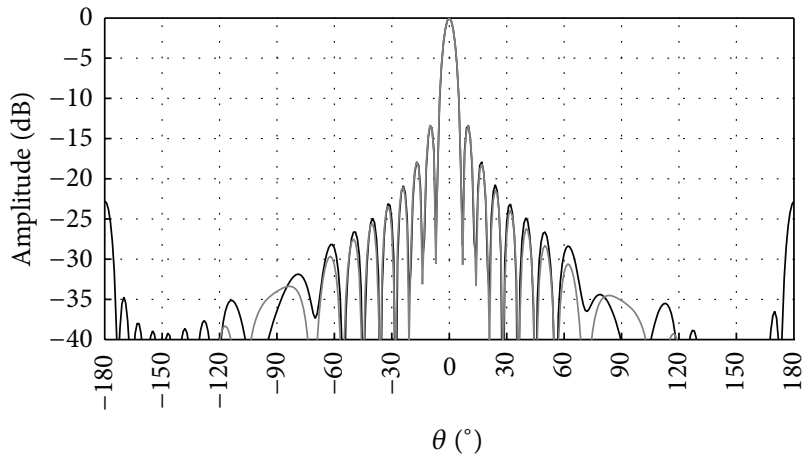

_ Full wave-CST Microwave
_ Analysis procedure-Matlab sofware

(a)

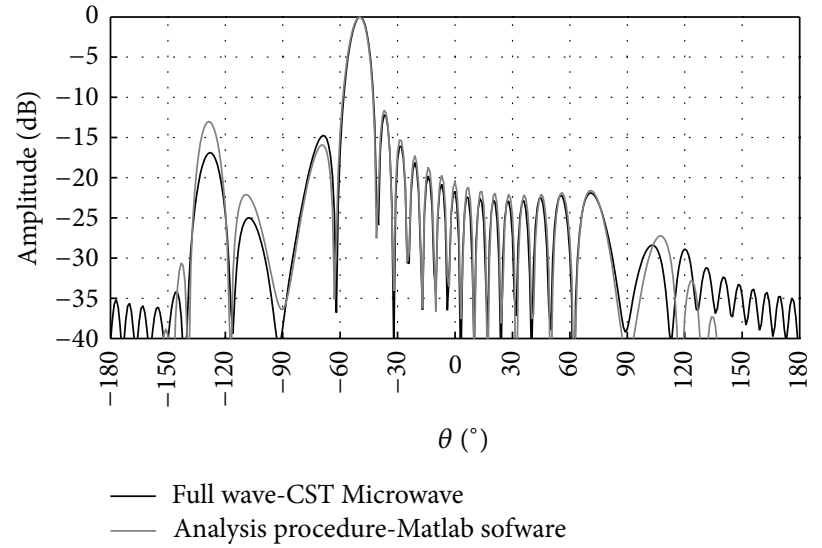

(b)

FIGURE 11: Comparison of normalized radiation patterns: (a) broadside radiation and (b) steering radiation at $-50^{\circ}$.

manufactured yet. The presented results are only theoretical and obtained using a full wave simulation (CST Microwave Studio). Therefore, the approach which consists of using simulation software is considered very reliable since several publications $[2,6]$ show that the measurement results are almost identical to those simulated considering the manufacturing imperfections. This novel design provides more flexibility and can be used in geographical coverage, beam forming, and beam steering applications.

\section{Conflict of Interests}

The authors declare that there is no conflict of interests regarding the publication of this paper.

\section{References}

[1] N. G. Alexopoulos and D. R. Jackson, "Fundamental superstrate effects on printed circuit antennas," IEEE Transactions on Antennas and Propagation, vol. AP-32, no. 8, pp. 807-816, 1984.

[2] A. P. Feresidis, G. Goussetis, S. Wang, and J. C. Vardaxoglou, "Artificial magnetic conductor surfaces and their application to low-profile high-gain planar antennas," IEEE Transactions on Antennas and Propagation, vol. 53, no. 1, pp. 209-215, 2005.

[3] H. Y. Yang and N. G. Alexopoulos, "Gain enhancement methods for printed circuit antennas through multiple superstrates," IEEE Transactions on Antennas and Propagation, vol. 35, no. 7, pp. 860-863, 1987.

[4] H. Boutayeb and T. A. Denidni, "Metallic cylindrical EBG structures with defects: Directivity analysis and design optimization," IEEE Transactions on Antennas and Propagation, vol. 55, no. 11, pp. 3356-3361, 2007.

[5] Y. J. Lee, J. Yeo, R. Mittra, and W. S. Park, "Design of a Frequency Selective Surface (FSS) type superstrate for dualband directivity enhancement of microstrip patch antennas," in Proceedings of the IEEE Antennas and Propagation Society International Symposium and USNC/URSI Meeting, pp. 2-5, Washington, DC, USA, July 2005.
[6] H. Yi and S.-W. Qu, "A novel dual-band circularly polarized antenna based on electromagnetic band-gap structure," IEEE Antennas and Wireless Propagation Letters, vol. 12, pp. 1149-1152, 2013.

[7] M. Hajj, E. Rodes, D. Serhal et al., "Metallic EBG sectoral antenna for base stations," in Proceedings of the 19th International Conference on Applied Electromagnetics and Communications (ICECom '07), pp. 1-4, September 2007.

[8] B. Jecko, M. Hajj, R. Chantalat, and M. Salah Toubet, "Antenne élémentaire et antenne réseau mono ou bidimensionnelle correspondante," PCT Patent PCT/EP2012/076509, University of Limoges, Limoges, France, 2013.

[9] M. Hajj, M. Salah Toubet, Y. Abdallah, R. Chantalat, and B. Jecko, "A novel beam scanning/directivity reconfigurable mEBG antenna array," Progress in Electromagnetics Research $C$, vol. 29, pp. 55-66, 2012. 

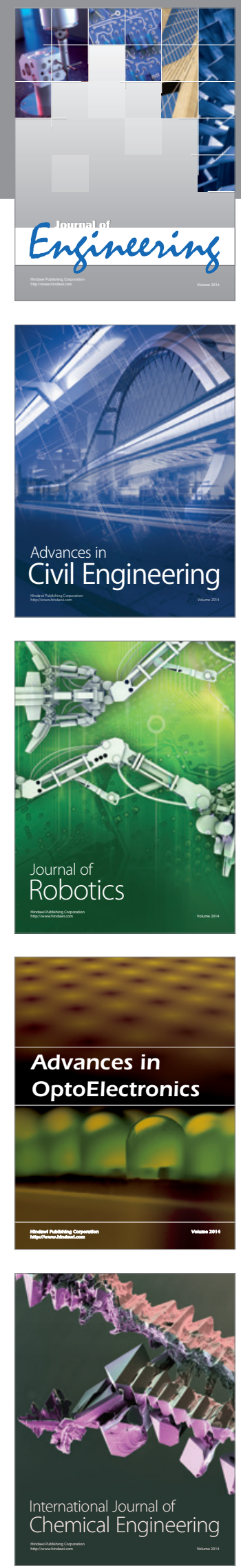

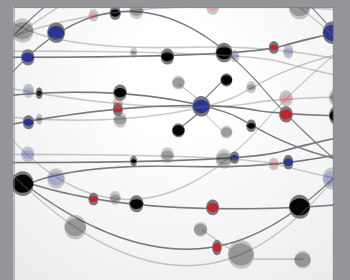

The Scientific World Journal
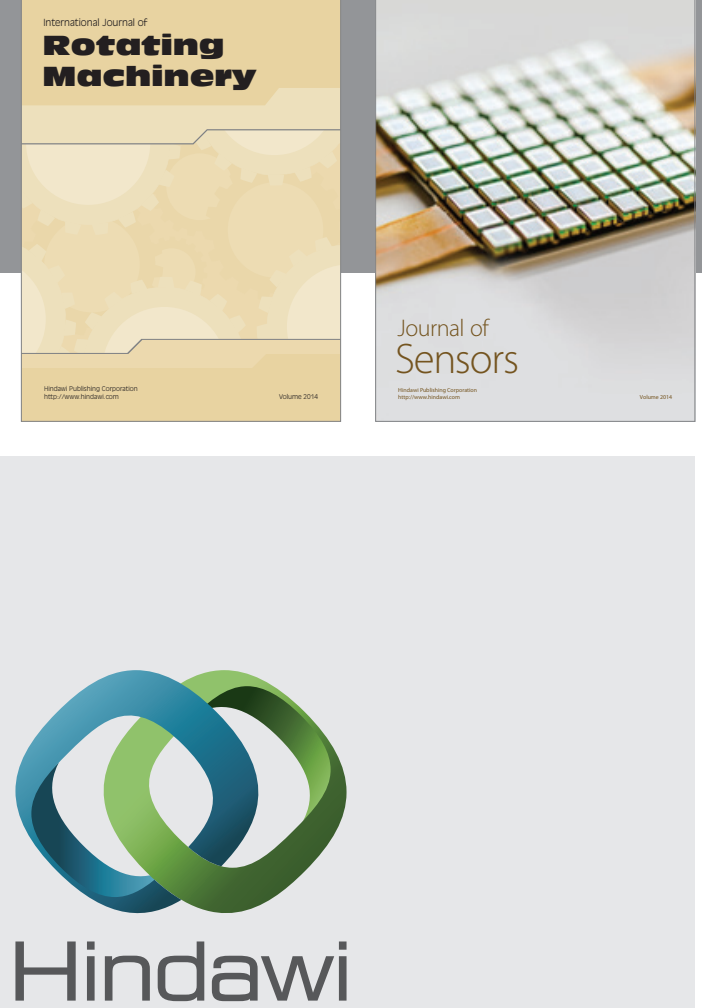

Submit your manuscripts at http://www.hindawi.com
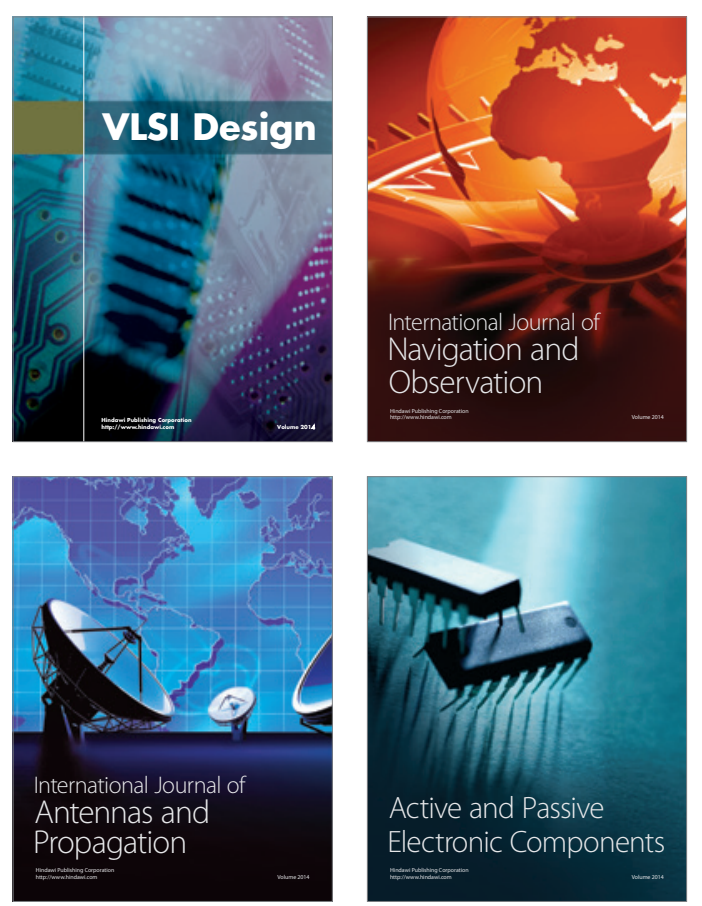
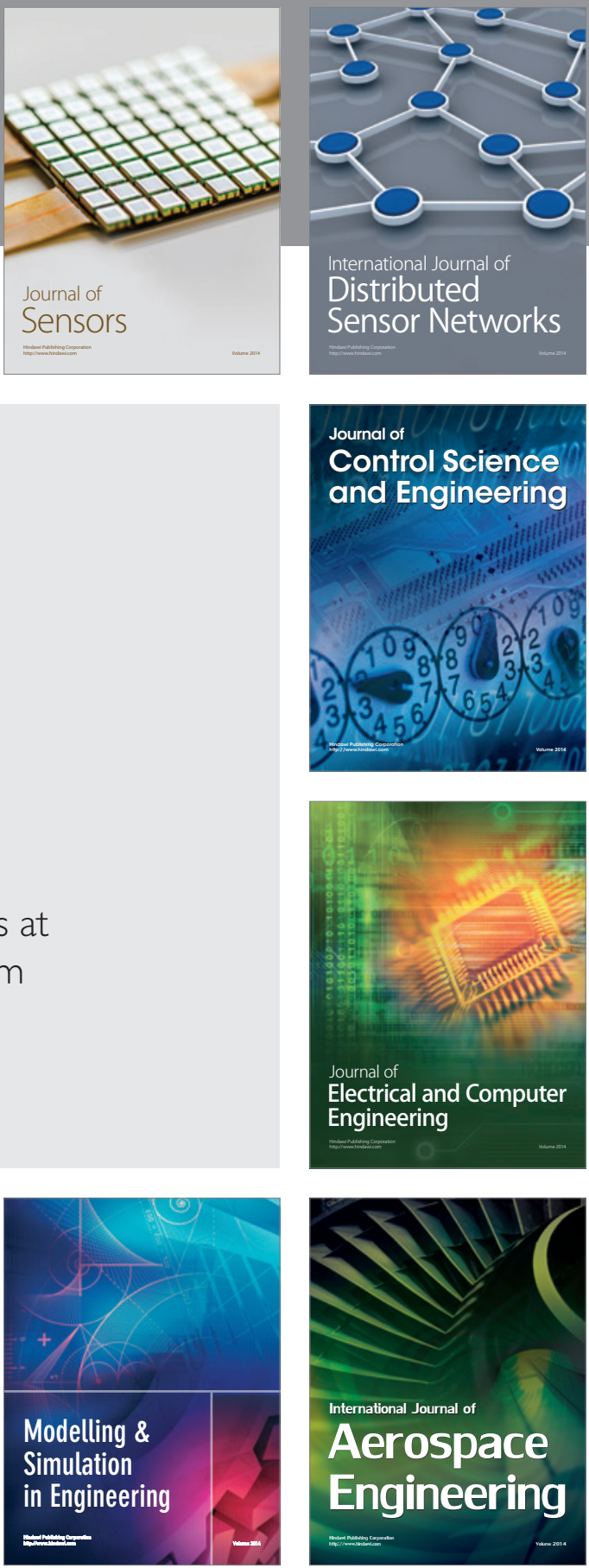

Journal of

Control Science

and Engineering
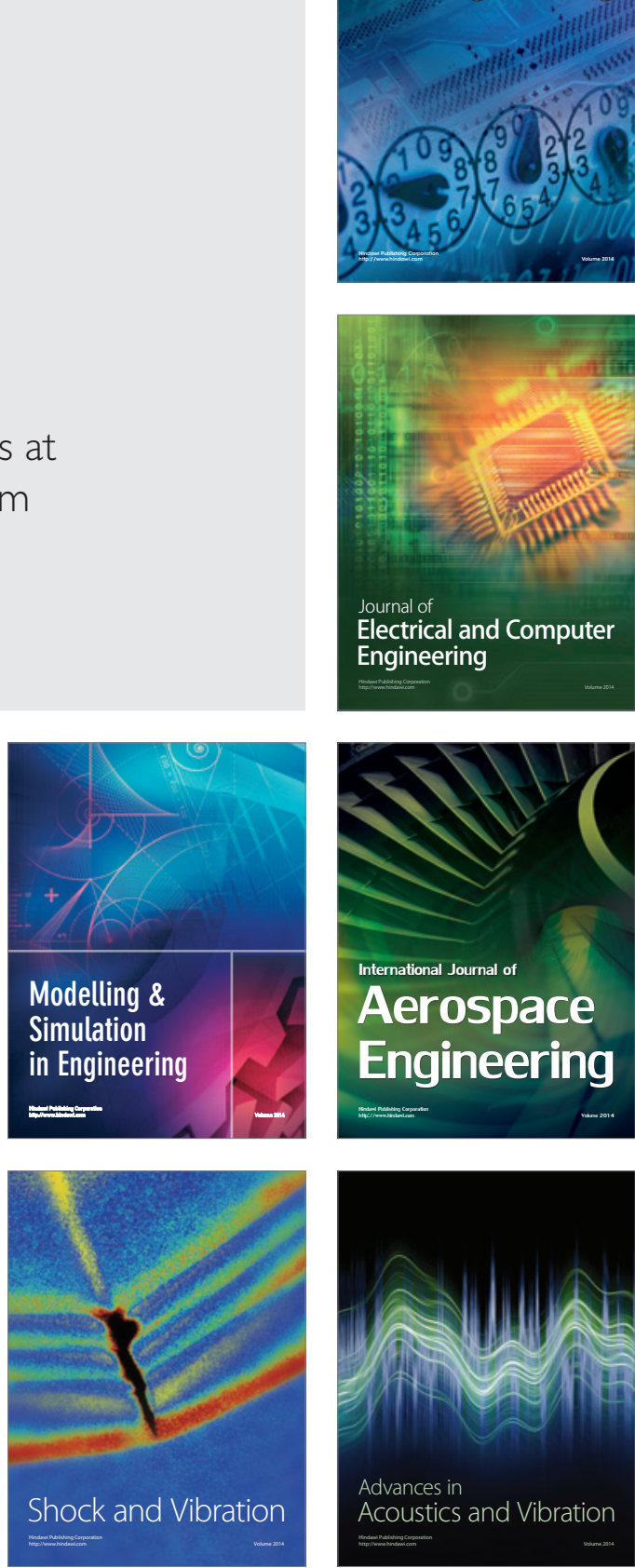